\title{
lonospheric Research in the European Auroral zone by Incoherent Scatter Techniques
}

\author{
T. Hagfors, Trondheim \\ (University of Trondheim)
}

Most of the early ground-based radio investigations of the ionosphere depended on the observation of $\mathrm{HF}$ waves reflected from those ionospheric heights where the refractive index of the wave becomes zero (cut-off region). This technique has provided considerable insight into the nature of the ionosphere but, the information obtained is limited in that only the ionosphere below the height of maximum electron density can give echoes and can be studied. The physical quantity which can be studied directly by this method is confined to electron density variation with height.

It was proposed in 1958 that a powerful beam of radiation at frequencies much higher than those which are totally reflected from the ionosphere could give rise to weak but detectable scattering from individual electrons and that this scattering could provide valuable information about the electron density at heights above that of maximum density. In addition to density it was thought that the Doppler broadening would be determined by the thermal motion of the electrons and that the electron temperature could also be deduced.

With the first experimental results it immediately became obvious that the total scattering cross section was approximately as predicted, but that the Doppler broadening was much less, thus making the detection of the scattering much easier than originally thought. The experimental evidence indicated and subsequent theoretical work confirmed that the Doppler broadening corresponds to the ionic motion. The shape of the Doppler spectrum turns out to be jointly controlled by the ion and the electron temperatures as well as by the ionic mass and composition. Clearly the incoherent scatter radar technique is a vastly more powerful tool for ionospheric research than originally anticipated.

Large and powerful observatories were built by organizations in the US, in Peru and in Puerto Rico for ionospheric research. Subsequently several mid-latitude observatories were built in the US, France and the UK and these too greatly contributed to our understanding of the ionosphere. Methods were developed for the study on a routine basis of electron density to several thousand kilometers height electron and ion temperatures and ion composition. The mean velocity of the plasma was measured and used for the determination of both electric fields as well as of the motions of the neutral atmosphere (which is much denser than the plasma). The observation of plasma oscillations by this technique revealed that photoelectrons released by solar radiation can travel from one hemisphere to the other along geomagnetic field lines.

The incoherent scatter radar technique has come to be regarded as the most powerful and comprehensive ground-based method for studying the upper atmosphere. The analysis of the returned signals allows a large number of parameters to be deduced These include: (1) electron density, (2) electron temperature, (3) positive ion temperature, (4) ion composition, (5) ion-neutral collision frequency, (6) line of sight plasma velocity, (7) the flux of suprathermal precipitating electrons and, in favourable cases, (8) the electric current density. From these direct measurements other parameters may be reliably derived, including: (9) electric field strength, (10) neutral air temperature, (11) electron-neutral collision frequency (12) neutral-air wind speed (13) neutral composition and (14) flux of heat energy.

Good precision can be obtained, with random errors of only a few percent in the best cases. The technique does not significantly perturb the medium and the results may therefore, be made substantially free from systematic errors. Continuous measurements may be made from $80 \mathrm{~km}$ to several thousand kilometers, although not all parameters may be measured over such a height range with a single mode of operation. No other ground-based technique produces so much information about the upper atmosphere. Many of the parameters cannot be measured from the ground in any other way.

Incoherent scatter is complementary to satellite and rocket measurements. Satellites provide good global coverage, but observations at any particular location are repeated only after long time intervals. Rockets are best suited to determining certain parameters such as the energy spectrum of precipitated particles, but they are so expensive to use, continuous measurements over long periods of time are out of the question.

\section{Application to the Polar Ionosphere}

The successful application of the incoherent scatter technique to the low and mid latitude ionosphere, suggested that the technique be applied to high latitudes. At these latitudes the ionosphere is much more complex because of magnetic coupling to the magnetosphere and because of widespread electron and proton precipitation. As a first step the XVI General Assembly of the International Union for Scientific Radio (URSI) held in Ottawa in 1969, passed resolutions recommending that an existing radar be moved to Alaska to test the method. Furthermore, it was recommended that second generation incoherent scatter installations be built in the American and the European sectors of the auroral zone.

The first phase of the recommendations was implemented by the transfer from Stanford, Cal. of a small, fully steerable radar operating at approximately $1300 \mathrm{MHz}$ into a $27 \mathrm{~m}$ parabolic dish to Chatanika, Alaska. This radar has been in operation for over three years and has, in spite of limited sensitivity, provided a spectacular amount of data on the auroral ionosphere. The operation of the Chatanika radar has also put to rest many of the original fears, that coherent echoes would be so prevalent as to render the incoherent scatter technique useless at auroral latitudes.

In response to the second part of the URSI recommendation the Upper Atmosphere Observatory (UAO) was planned by US and Canadian groups for the North American Sector and the European Incoherent SCATter Observatory (EISCAT) for the European Sector.

\section{EISCAT, Facility and Operation}

The EISCAT Scientific Association is jointly operated by research councils in the Federal Republic of Germany, Finland, France, Norway, Sweden and the UK. The observatory will consist of stations in Tromsø, Norway; 
Kiruna, Sweden and Sodankylä, Finland. The transmitters will operate at two frequencies, $224 \mathrm{MHz}$ (VHF) and 933.5 $\mathrm{MHz}$ (UHF) from Tromsø at power levels of $250 \mathrm{~kW}$ average and several MW peak. At VHF the transmitter and receiver will operate on the same antenna. At UHF there will be receivers also at Kiruna and at Sodankylä.

The VHF system which will be the more sensitive, will be able to scan in a plane in the magnetic meridian. It is expected that observations with this system may be made to heights of several thousand kilometers and that the system will be sensitive to a variety of plasma oscillations or instabilities caused by precipitating particles and strong electric fields associated with auroral activity. Plasma motions will, however, only be observable along the direction of the antenna beam.

The UHF system expected to be in operation in $78 / 79$, will transmit from a fixed antenna and will be less sensitive. However, the receiving stations at Kiruna and Sodankylä will view the scattering volume from different angles with narrow antenna beams thereby, permitting by the Doppler effect, three components of the plasma velocity to be measured simultaneously, with height resolution of better than $5 \mathrm{~km}$ at most heights.

The observatory will be operated from a small headquarter in Kiruna through a system of contracts with the University of Troms $ø$ and with the Geophysical Institutes of Kiruna and Sodankylä. The observatory will be available for experimentation to any scientist within the member countries on the basis of proposals. It is also foreseen that scientists from nonmember countries may use the observatory by special arrangement.

\section{Scientific Problems for EISCAT}

It has been predicted that there is a steady outflow of plasma along the magnetic field lines in the polar regions, the so-called polar wind. It is expected that EISCAT will be able to measure this important geophysical phenomenon directly and continuously. Measurements of the plasma motion at lower altitudes will give direct evidence of thermospheric winds which influence the behaviour of the ionosphere in a major way.

The measurement of plasma drift, perpendicular to the magnetic field lines, can be used to determine the electric fields. Due to the coupling by the magnetic field between the ionosphere and the magnetosphere, the distribution of electric fields will provide information regarding the large scale circulation of plasma in the magnetosphere. The ESA GEOS satellite (EN April 76) will lie on almost the same magnetic field line as EISCAT, so if GEOS is still in operation when EISCAT starts up, a comparison of electric field and other data, will undoubtedly increase our understanding of the ionosphere magnetosphere coupling.

The electric fields in the ionosphere produce currents, particularly at auroral latitudes. These currents which may be measured directly by EISCAT contribute to geomagnetic variations at ground level. Any information on these currents is of great value to geomagnetism.

The input of energy by particles and electric currents at high latitudes constitutes a major heat source for the thermosphere, rivalling the one due to solar ionizing radiation. The temperature measurements of EISCAT may be of vital importance to these energy balance studies.

Plasma oscillations which can be directly measured by the EISCAT facility are excited by suprathermal electrons and thus direct observation of the low energy tail of the precipitating electron flux will be possible.

Electron density observations in the $D$ and the E-regions with short timeresolution will also indicate the flux and spectrum of precipitating electrons and may also give the effective recombination coefficients at these heights. It is also expected that studies can be made of the relation between stratospheric warning and Dregion effects, seasonal and storminduced variations in F-region composition and of gravity waves and travelling ionospheric disturbances.

\section{Other Types of Observation}

Egeland in a companion article describes a number of other experiments, ground based as well as rocket born, all designed to study phenomena identical to, or closely related to those which will be investigated by EISCAT. In addition to the instrumentation already available in Northern Scandinavia, it appears as if there will be several new experiments planned in conjunction with EISCAT. These will include, amongst others, a set of oblique looking coherent type backscatter radars as well as high power HF transmitters designed for the artificial modification of the ionospheric layers above the EISCAT facility.

lonospheric and magnetospheric physicists in Europe are, therefore, looking forward to the new facility with considerable anticipation.

\section{NEW BOORS The Hydrogen Bond} Recent Developments in
Theory and Experiment

(in 3 volumes)

edited by P. SCHUSTER, Universität Wien, G. ZUNDEL, Universität München, and C. SANDORFY, Université de Montréal.

\section{6. about 1520 pages \\ Price: US $\$ 179.95 /$ Dfl. 450.00 per 3-vol. set}

This three-volume work provides, for the first time, a truly comprehensive survey of the field, comprising hitherto unpublished original work, together with relevant material previously scattered throughout the literature. The twenty-nine chapters contained in this work are each written by a specialist in the particular discipline. Collectively, they cover the various approaches towards an understanding of hydrogen bonds.

\section{Volume I - Theory.}

Contains an introduction and six chapters reflecting the present state of theory. Starting from energy surfaces of hydrogen bonds, calculations of vibrational spectra, proton motion, dynamics of proton networks and hydrogen bond statistics are treated extensively.

\section{Volume II - Structure and Spectro-} scopy.

Experimental results on structure and stereochemistry of hydrogen bonds in crystals and liquids are described. Five chapters provide detailed insight into the current state of infrared and long wave length vibrational spectroscopy. Nuclear magnetic resonance studies are reported, especially results obtained from solids.

\section{Volume III - Dynamics, Thermo-} dynamics and Special Systems.

Presents a survey of experimental results on hydrogen bond dynamics, as studied by incoherent neutron scattering, dielectric methods, spinlattice and chemical relaxation techniques. Two chapters deal with hydrogen bonded ferroelectrics and the subsequent contributions report thermodynamic results and their correlation with spectral data. The last chapters present results on hydrogen bonding in surface chemistry, water and ice, as well as in studies by the matrix isolation technique.

\section{NORTH-HOLLAND PUBLISHING CO.}

(1)

